\title{
A STUDY OF THE ELECTROMAGNETIC FIELD FROM THE MCSEM DIPOLE SOURCE IN AN ANISOTROPIC LAYERED EARTH
}

\author{
Walleson Gomes dos Santos ${ }^{1,2}$ and Cícero Roberto Teixeira Régis ${ }^{1,2}$
}

\begin{abstract}
This paper studies the electromagnetic field from a horizontal electrical dipole inside a layered earth model with TIV anisotropy, including a visualization of the geometric patterns of the field through the medium. The objective here is to present a detailed formulation of the problem, as an aid to those who have an interest in modeling data from the Marine Controlled-Source ElectroMagnetic method - mCSEM, but find it hard to follow the usually abridged, often incomplete, descriptions found in the technical literature. We present a detailed vector potential formulation, with a semi-analytical solution, that allows the calculation of the fields with the source located in a finite thickness ocean layer over N-layered earth models. As an application, we use the implemented solution to study the geometrical distribution of the electric field generated by the dipole source in anisotropic layered media.
\end{abstract}

Keywords: mCSEM, TIV electrical anisotropy, 1D electromagnetic modeling.

RESUMO. Este artigo estuda o campo eletromagnético de um dipolo elétrico horizontal no interior de um modelo estratificado com anisotropia TIV, incluindo uma visualização da geometria das linhas de campo através do meio. 0 objetivo é apresentar a formulação detalhada do problema, para aqueles que têm interesse na modelagem de dados do método eletromagnético de fonte controlada marinho - mCSEM, mas encontram dificuldades em seguir as descrições geralmente muito resumidas, diversas vezes incompletas, na literatura técnica. Apresentamos uma formulação detalhada em termos do potencial vetorial, com uma solução semi-analítica que permite 0 cálculo dos campos com a fonte localizada em um oceano de espessura finita sobre uma terra estratificada com N camadas. Como aplicações, usamos a solução implementada para estudar a distribuição geométrica do campo elétrico gerado pelo dipolo fonte em meios estratificados anisotrópicos.

Palavras-chave: mCSEM, anisotropia elétrica TIV, modelagem eletromagnética 1D.

\footnotetext{
1 Universidade Federal do Pará, Programa de Pós-Graduação em Geofísica - CPGf, Rua Augusto Corrêa, 01, Guamá, 66075-110 Belém, PA, Brazil. Phone: +55(91) 3201-7692 - E-mails: walleson_santos@yahoo.com.br; cicero@ufpa.br

2 Instituto Nacional de Ciência e Tecnologia de Geofísica do Petróleo - INCT-GP, Phone: +55(71) 3283-8551
} 


\section{INTRODUCTION}

The electromagnetic field from the marine CSEM dipolar antenna is generated by an electric current on the order of $1000 \mathrm{~A}$, or higher (Constable \& Srnka, 2007). The current flows through the water and the sediments below, and its variations generate electromagnetic induction that helps the signal to be measured at distances several kilometers away from the source. Both galvanic and inductive effects contribute to the diffusion of the EM field.

In the interpretation of marine CSEM data the environment under the sea floor can be considered as electrically isotropic in many cases. However, it is well documented that sequences of sediment layers frequently found in oil and gas exploration are perceived as a homogeneous medium with effective electrical anisotropy (Tompkins, 2005). The effects of anisotropy must be considered in the interpretation, especially the fact that the vertical component of the conductivity has a stronger influence on the electric field measured by the inline receivers than it has on that measured by the crossline receivers (Lu \& Xia, 2007).

In order to build a clear understanding of these phenomena, it is important to know how the geometry of the electric field and the current density field lines are affected by the geoelectric structures of the sea plus sediments environment, because galvanic effects have a dominant role in the field diffusion in this highly conductive medium. In the mathematically simplest possible case, the electric dipole source would be in an infinite homogeneous conductive medium, and the electric field would simply follow the pattern of a dipole field, with its characteristic symmetry. In more complex environments, the fields and the electric current flow are altered by the geometry, as well as by the physical properties of the different conductivity zones in the media. All these effects, in turn, have an impact on the components that are measured in the mCSEM method.

The problem of calculating the fields from an electric dipole in layered anisotropic models has been treated by several authors. Xiong (1989) presents a vector potential solution following the traditional recursive algorithm in terms of reflection coefficients (Wait, 1981, chapter 11), much like the method followed here. However, the presentation in his short note is indeed extremely short, and does not give many details of the calculation. The work is not helped by the fact that the plots with the results for the halfspace model are not correct, and do not correspond to the true solution for that model.

Løseth \& Ursin (2007) presented a general formulation for electric and magnetic dipole sources, based on propagation matrices. Their method has applications to a number of different geophysical sources, but it demands the evaluation of the double inverse Fourier transform, which is a heavy numerical task.
A classical procedure in finite element solutions of $2 \mathrm{D}$ or $3 \mathrm{D}$ problems is to build models formed by a layered background inside which are placed target bodies. The fields inside the $1 \mathrm{D}$ layered media are used in the source term in the differential equations, which avoids the trouble of representing the point sources in the discretized media represented by the finite-element meshes. Several applications of finite-elements to the MCSEM problem in anisotropic environments can be found. Kong et al. (2008) presented one application, using a secondary field formulation, which they claim to be inventing (in 2008!). These authors do not show a detailed development of the primary field calculations. They present a solution using non-standard reflection coefficients associated with the air/water interface and they do not present the expressions for the coefficients associated with the sea bed interface, citing Kong (1990) as the reference for the method to calculate those expressions. The extremely short presentation of part of the solution makes this reference particularly hard to follow, if one is to reproduce the results.

Another 2.5D finite-element solution to the anisotropic problem, using primary and secondary fields is given by Ramananjaona \& MacGregor (2010), however their primary field are calculated in isotropic half-space models, the anisotropy being introduced only in the 2D part of the complete models. Li \& Dai (2011) have modeled the more general case of dipping anisotropy in yet another 2.5D formulation using an isotropic layered background to generate their primary fields. This use of isotropic $1 \mathrm{D}$ models is sufficient to generate the primary fields, leaving the anisotropy to be included in the $2 \mathrm{D}$ parts of the models. However, this also implies a need for a greater number of nodes in the meshes if an anisotropic layer is to be modeled as a long $2 \mathrm{D}$ body. Therefore, it is preferable that the primary fields be calculated in anisotropic layered models.

In this paper we study a detailed and complete formulation of the problem of calculating the frequency domain fields from an electric dipole in layered earth models, to simulate mCSEM data. The method uses the vector potential associated with the magnetic field, and the problem is solved in the Fourier domain. The use of the vector potential allows the separation of the fields in TE and TM contributions, and the symmetry of the $1 \mathrm{D}$ problem allows the inverse double Fourier transforms to be changed into single integral Hankel transforms, which can be efficiently solved numerically using digital filters.

The main objective of this paper is to offer a solution that can be followed and implemented by anyone who's interested in this problem, particularly if they intend to use the $1 \mathrm{D}$ solution as primary fields in a more general formulation. 
As an application of the method, we also present a study of how the variations in conductivity in a layered earth affect and deform the electric field lines away from the dipole field configuration, and how the presence of anisotropic layers impose further changes in that geometry. The visualization of the electric field allows us to analyse the effect of the anisotropy inside the layers, and its influence on the mCSEM data.

\section{MATHEMATICAL FORMULATION OF THE PROBLEM}

We follow the classical procedure of first calculating the fields from the source in an unbounded homogeneous medium, and then using these as incident fields on the layered medium. The formulation builds the differential equations in terms of the vector potential associated with the magnetic field through the rotational operator. When the fields in the unbounded medium are calculated in the domain of the Fourier transform, with respect to the horizontal $x$ and $y$ coordinates, the result is a spectral decomposition in terms of plane wave solutions. From these, a plane wave field inside the layered medium is calculated. The final steps are the operations to return to the electromagnetic field from the potential's solution and to perform the inverse transform to generate the solutions in the spatial coordinates.

\section{Unbounded medium}

As always, start with Maxwell's equations. At the frequency domain, in conductive media, and in the quasi-static regime $(\sigma \gg$ $\left.\omega \epsilon_{0}\right)$ :

$$
\begin{aligned}
\nabla \cdot E & =0, \\
\nabla \cdot H & =0, \\
\nabla \times E+i \omega \mu H & =0, \\
\nabla \times H-\sigma E & =I(\omega) d s \delta(x) \delta(y) \delta\left(z-z_{t}\right) .
\end{aligned}
$$

The term $I(\omega) d s \delta(x) \delta(y) \delta\left(z-z_{t}\right)$ represents the current density function of the dipole source transmitter located at $\left(0,0, z_{t}\right)$. For an $x$ oriented dipole, $d s=\left(d s_{x}, 0,0\right)$. $I(\omega) d s_{x}$ represents the dipole moment.

For transversely isotropic layered media with vertical simmetry axis (TIV), the conductivity tensor is

$$
\sigma=\left(\begin{array}{ccc}
\sigma_{h} & 0 & 0 \\
0 & \sigma_{h} & 0 \\
0 & 0 & \sigma_{v}
\end{array}\right) .
$$

Define the vector potential $A$ so that

$$
H=\nabla \times A
$$

Then, from Faraday law (equation 3):

$$
\begin{array}{r}
\nabla \times(E+i \omega \mu A)=0 ; \\
E=-i \omega \mu A-\nabla U,
\end{array}
$$

where $U$ is a scalar field.

Now write Ampère's law (4) in terms of the vector potential by using its relations with the fields (equations 6 and 8):

$$
\begin{gathered}
\nabla \times \nabla \times A+\sigma(i \omega \mu A+\nabla U) \\
=I(w) d s \delta(x) \delta(y) \delta\left(z-z_{t}\right) .
\end{gathered}
$$

The vector identity $\nabla \times \nabla \times A=-\nabla^{2} A+\nabla(\nabla \cdot A)$ makes

$$
\begin{gathered}
-\nabla^{2} A+\nabla(\nabla \cdot A)+\sigma \nabla U+i \omega \mu \sigma A \\
=I(w) d s \delta(x) \delta(y) \delta\left(z-z_{t}\right) .
\end{gathered}
$$

This is a system of three equations:

$$
\begin{aligned}
- & \nabla^{2} A_{x}+\frac{\partial}{\partial x}(\nabla \cdot A)+\sigma_{h} \frac{\partial U}{\partial x} \\
& +i \omega \mu \sigma_{h} A_{x}=I(w) d s_{x} \delta(x) \delta(y) \delta\left(z-z_{t}\right), \\
- & \nabla^{2} A_{y}+\frac{\partial}{\partial y}(\nabla \cdot A)+\sigma_{h} \frac{\partial U}{\partial y} \\
& +i \omega \mu \sigma_{h} A_{y}=0, \\
- & \nabla^{2} A_{z}+\frac{\partial}{\partial z}(\nabla \cdot A)+\sigma_{v} \frac{\partial U}{\partial z} \\
& +i \omega \mu \sigma_{v} A_{z}=0 .
\end{aligned}
$$

Following the procedure described in (Kaufman \& Dashevsky, 2003), a convenient configuration for the vector potential is $A=\left(A_{x}, 0, A_{z}\right)$, which reduces the number of components while still generating the complete magnetic field. A good gauge condition is, then

$$
\nabla \cdot A=-\sigma_{h} U
$$

These choices lead to the system of two coupled differential equations on the components of $A$ :

$$
\begin{aligned}
\nabla^{2} A_{x}+k_{h}^{2} A_{x} & =-I(\omega) d s_{x} \delta(x) \delta(y) \delta\left(z-z_{t}\right), \\
\nabla^{2} A_{z}+k_{v}^{2} A_{z} & =\left(1-\frac{\sigma_{v}}{\sigma_{h}}\right) \frac{\partial}{\partial z} \nabla \cdot A
\end{aligned}
$$

where two wave numbers are defined: one associated with the horizontal conductivity $k_{h}$, and the other associated with the vertical conductivity $k_{v}$. In order to simplify notation we also define 
two more functions $u$ and $v$ as follows:

$$
\begin{aligned}
& k_{h}^{2}=-i \omega \mu \sigma_{h}, \\
& k_{v}^{2}=-i \omega \mu \sigma_{v}, \\
& u^{2}=k_{x}^{2}+k_{y}^{2}-k_{h}^{2}, \\
& v^{2}=k_{x}^{2}+k_{y}^{2}-k_{v}^{2} .
\end{aligned}
$$

Here we also follow the usual definition for the coefficient of anisotropy $\lambda$ (Spies \& Frischknecht, 1987, page 350, eq. 47):

$$
\lambda^{2}=\frac{\sigma_{h}}{\sigma_{v}}
$$

The gauge condition also allows us to write the electric field as

$$
E=-i \omega \mu A+\frac{1}{\sigma_{h}} \nabla(\nabla \cdot A) .
$$

The solutions to these equations in the Fourier domain $\left(k_{x}, k_{y}, z\right)$ allow for a representation in terms of a plane wave spectral decomposition. The equation with the source term (15) yields

$$
\hat{\hat{A}}_{x}\left(k_{x}, k_{y}, z\right)=\left\{\begin{array}{c}
I(\omega) d s_{x} \frac{e^{-u\left(z-z_{t}\right)}}{2 u}, \\
\left(z-z_{t}\right) \geq 0, \\
I(\omega) d s_{x} \frac{e^{u\left(z-z_{t}\right)}}{2 u}, \\
\left(z-z_{t}\right) \leq 0,
\end{array}\right.
$$

From the equation for $A_{z}$ (16) we get

$$
\begin{aligned}
\frac{\partial^{2} A_{z}}{\partial x^{2}} & +\frac{\partial^{2} A_{z}}{\partial y^{2}}+\frac{1}{\lambda^{2}} \frac{\partial^{2} A_{z}}{\partial z^{2}}+k_{v}^{2} A_{z} \\
& =\left(1-\frac{1}{\lambda^{2}}\right) \frac{\partial^{2} A_{x}}{\partial x \partial z}
\end{aligned}
$$

which leads to the spectral domain $\left(k_{x}, k_{y}, z\right)$ representation:

$$
\frac{\partial^{2} \hat{\hat{A}}_{z}}{\partial z^{2}}-\lambda^{2} v^{2} \hat{\hat{A}}_{z}=\left(\lambda^{2}-1\right) i k_{x} \frac{\partial \hat{\hat{A}}_{x}}{\partial z} .
$$

By applying the expression for $A_{x}$ (23) to the equation for $A_{z}$ (25), taking the derivative $\partial \hat{\hat{A}}_{x} / \partial z$, and making $k_{r}^{2}=$ $k_{x}^{2}+k_{y}^{2}$, the solution is the sum of the general solution for the homogeneous equation with a particular solution for the non homogeneous equation:

$$
\begin{gathered}
\hat{\hat{A}}_{z}\left(k_{x}, k_{y}, z\right)= \\
\left\{\begin{array}{l}
\frac{i k_{x} I(\omega) d s_{x}}{2 k_{r}^{2}}\left(e^{-u\left(z-z_{t}\right)}-e^{-\lambda v\left(z-z_{t}\right)}\right), \\
\left(z-z_{t}\right) \geq 0 \\
-\frac{i k_{x} I(\omega) d s_{x}}{2 k_{r}^{2}}\left(e^{u\left(z-z_{t}\right)}-e^{\lambda v\left(z-z_{t}\right)}\right), \\
\left(z-z_{t}\right) \leq 0
\end{array}\right.
\end{gathered}
$$

\section{Layered medium}

The expressions for $\hat{\hat{A}}_{x}$ and $\hat{\hat{A}}_{z}$ (equations 23 and 26) have the form of the $z$ dependent part of plane wave solutions with incidence angles that are a function of $k_{r}$ (Ward \& Hohmann, 1987). Therefore, it is possible to write the solution inside the layers as combinations of downgoing and upgoing plane wave fields (of the vector potential field $\hat{\hat{A}}$ ), in terms of transmission and reflection coefficients. There is one more term in the equations for the $\hat{\hat{A}}_{z}$ component in the unbounded medium, as compared to the isotropic case, because now there are two wave numbers to be considered.

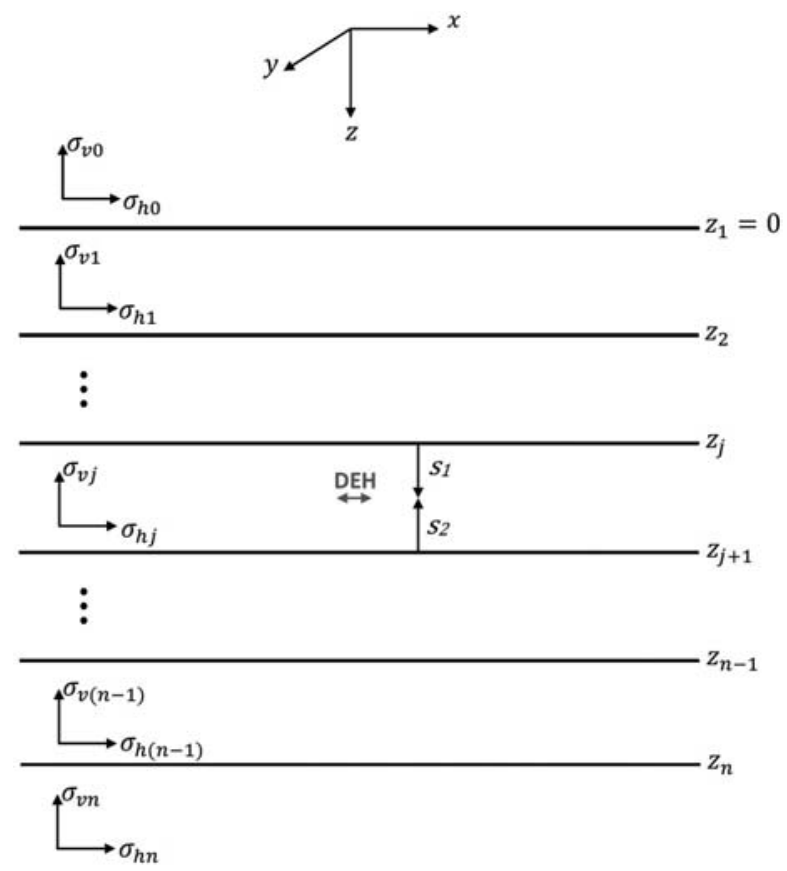

Figure 1 - Anisotropic layered model.

The general model for the layered medium is illustrated in Figure 1. In mCSEM problems, the top infinite half-space, with index 0 , is the air. Each layer $i$ has thickness $h_{i}$. Its physical properties are used in the definition of its intrinsic impedance $\mathcal{Z}_{i}$ and intrinsic admittance $\mathcal{Y}_{i}$, in the $\left(k_{x}, k_{y}, z\right)$ domain:

$$
\begin{gathered}
\mathcal{Z}_{i}=\frac{\lambda_{i} v_{i}}{\sigma_{h_{i}}} \\
\mathcal{Y}_{i}=\frac{u_{i}}{i \omega \mu_{i}}
\end{gathered}
$$

In this general solution, it is admitted that there are layers above the source, which is located in layer $j$. Now we write the solutions for the vector potential inside this layer, in the next layer beneath it $(j+1)$, in every arbitrary layer below it $(j+k)$, and in the infinite half-space at the bottom $(n)$ : 


$$
\begin{gathered}
\hat{\hat{A}}_{x_{(-)}}^{(j)}=A_{x j}\left[\begin{array}{c}
e^{u_{j}\left(z-z_{j}\right)}+R_{x}^{(j)} e^{-u_{j}\left(z-z_{j}\right)} \\
+R_{x}^{(j+1)} e^{u_{j}\left(z-z_{j}-2 s_{2}\right)}
\end{array}\right], \\
\hat{\hat{A}}_{x_{(+)}}^{(j)}=A_{x j}\left[\begin{array}{c}
e^{-u_{j}\left(z-z_{j}-2 s_{1}\right)}+R_{x}^{(j)} e^{-u_{j}\left(z-z_{j}\right)} \\
+R_{x}^{(j+1)} e^{u_{j}\left(z-z_{j}-2 s_{2}\right)}
\end{array}\right], \\
\hat{\hat{A}}_{x}^{(j+1)}=A_{x(j+1)}\left[\begin{array}{c}
e^{-u_{j+1}\left(z-z_{j+1}\right)} \\
+R_{x}^{(j+2)} e^{u_{j+1}\left(z-z_{j+1}-2 h_{j+1}\right)}
\end{array}\right], \\
\hat{\hat{A}}_{x}^{(j+k)=A_{x(j+k)}}\left[\begin{array}{c}
e^{-u_{j+k}\left(z-z_{j+k}\right)} \\
+R_{x}^{(j+k+1)} e^{u_{j+k}\left(z-z_{j+k}-2 h_{j+k}\right)}
\end{array}\right], \\
\hat{\hat{A}}_{x}^{(n)}=A_{x n} e^{-u_{n}\left(z-z_{n}\right)} .
\end{gathered}
$$

where

$$
A_{x j}=\frac{I(\omega) d s_{x} e^{-u_{j} s_{1}}}{2 u_{j}}
$$

The (-) and (+) subscripts mean that the layer $j$ is, respectively, above or below the one with the dipole source.

The component $\hat{\hat{A}}_{z}$ is determined by using the expressions for $\hat{\hat{A}}_{x}(29)$ in the differential equation relating both components (25). Again, the solution is the sum of the general solution for the homogeneous equation (terms in $\mathcal{A}$, in the following expressions) with a particular solution for the non homogeneous equation; for a somewhat more compact expression, these solutions can be written in terms of the same derivatives $\partial \hat{\hat{A}}_{x}^{(i)} / \partial z$, as they appear in the final form, which is composed of the exponential functions:

$$
\begin{aligned}
& \hat{\hat{A}}_{z_{(-)}}^{(j)}=\frac{-i k_{x}}{k_{r}^{2}} \frac{\partial \hat{\hat{A}}_{x_{(-)}}^{(j)}+\mathcal{A}_{v_{(-)}}^{(j)}}{\partial z} \\
& \hat{\hat{A}}_{z_{(+)}(j)}=\frac{-i k_{x}}{k_{r}^{2}} \frac{\partial \hat{\hat{A}}_{x_{(+)}}^{(j)}+\mathcal{A}_{v_{(+)}}^{(j)},}{\partial z} \\
& \hat{\hat{A}}_{z}^{(j+1)}=\frac{-i k_{x}}{k_{r}^{2}} \frac{\partial \hat{\hat{A}}_{x}^{(j+1)}}{\partial z}+\mathcal{A}_{v}^{(j+1)}, \\
& \hat{\hat{A}}_{z}^{(j+k)}=\frac{-i k_{x}}{k_{r}^{2}} \frac{\partial \hat{\hat{A}}_{x}^{(j+k)}}{\partial z}+\mathcal{A}_{v}^{(j+k)}, \\
& \hat{\hat{A}}_{z}^{(n)}=\frac{-i k_{x}}{k_{r}^{2}} \frac{\partial \hat{\hat{A}}_{x}^{(n)}}{\partial z}+\mathcal{A}_{v}^{(n)},
\end{aligned}
$$


with,

$$
\begin{gathered}
\mathcal{A}_{v_{(-)}}^{(j)}=A_{z j}\left[\begin{array}{c}
e^{\lambda_{j} v_{j}\left(z-z_{j}\right)}+R_{z}^{(j)} e^{-\lambda_{j} v_{j}\left(z-z_{j}\right)} \\
-R_{z}^{(j+1)} e^{\lambda_{j} v_{j}\left(z-z_{j}-2 s_{2}\right)}
\end{array}\right], \\
\mathcal{A}_{v_{(+)}}^{(j)}=-A_{z j}\left[\begin{array}{c}
e^{-\lambda_{j} v_{j}\left(z-z_{j}-2 s_{1}\right)}-R_{z}^{(j)} e^{-\lambda_{j} v_{j}\left(z-z_{j}\right)} \\
+R_{z}^{(j+1)} e^{\lambda_{j} v_{j}\left(z-z_{j}-2 s_{2}\right)}
\end{array}\right], \\
\mathcal{A}_{v}^{(j+1)}=A_{z(j+1)}\left[\begin{array}{c}
-e^{-\lambda_{j+1} v_{j+1}\left(z-z_{j+1}\right)} \\
-R_{z}^{(j+2)} e^{\lambda_{j+1} v_{j+1}\left(z-z_{j+1}-2 h_{j+1}\right)}
\end{array}\right], \\
\mathcal{A}_{v}^{(j+k)}=A_{z(j+k)}\left[\begin{array}{c}
-e^{-\lambda_{j+k} v_{j+k}\left(z-z_{j+k}\right)} \\
-R_{z}^{(j+k+1)} e^{\lambda_{j+k} v_{j+1}\left(z-z_{j+k}-2 h_{j+k}\right)}
\end{array}\right], \\
\mathcal{A}_{z}^{(n)}=-A_{z n} e^{-\lambda_{n} v_{n}\left(z-z_{n}\right)} .
\end{gathered}
$$

where

$$
A_{z j}=\frac{I(\omega) d s_{x} i k_{x} e^{-\lambda_{j} v_{j} s_{1}}}{2 k_{r}^{2}} .
$$

The continuity conditions for the tangential components of the EM field lead to (Kaufman \& Dashevsky, 2003, page 620)

$$
\begin{gathered}
\hat{\hat{A}}_{x}^{(i)}=\left.\hat{\hat{A}}_{x}^{(i+1)}\right|_{z=z_{(i+1)}} \quad \text { and } \quad \frac{\partial \hat{\hat{A}}_{x}^{(i)}}{\partial z}=\left.\frac{\partial \hat{\hat{A}}_{x}^{(i+1)}}{\partial z}\right|_{z=z_{(i+1)}}, \\
\mathcal{A}_{v}^{(i)}=\left.\mathcal{A}_{v}^{(i+1)}\right|_{z=z_{(i+1)}} \text { and } \quad \frac{1}{\sigma_{h_{i}}} \frac{\partial \mathcal{A}_{v}^{(i)}}{\partial z}=\left.\frac{1}{\sigma_{h_{i+1}}} \frac{\partial \mathcal{A}_{v}^{(i+1)}}{\partial z}\right|_{z=z_{(i+1)}} .
\end{gathered}
$$

The transmission coefficients $A_{x(j+1)}, A_{x(j+k)}$, and $A_{x n}$, at the interfaces $j+1, j+k$, and $n$, are determined using the continuity condition expressions for $\hat{\hat{A}}_{x}(32)$ :

$$
\begin{aligned}
A_{x(j+1)}= & \frac{2 A_{x j} u_{j} R_{x}^{(j+1)} e^{u_{j}\left(s_{1}-s_{2}\right)}}{\left(u_{j}-u_{j+1}\right)+\left(u_{j}+u_{j+1}\right) R_{x}^{(j+2)} e^{-2 u_{j+1} h_{j+1}}}, \\
A_{x(i+2)}= & \frac{A_{x(i+1)}\left(1+R_{x}^{(i+2)}\right) e^{-u_{i+1} h_{i+1}}}{1+R_{x}^{(i-3)} e^{-2 u_{i+2} h_{i+2}}} \\
& i=j, j+1, \ldots, j+k-2, \\
A_{x n}= & A_{x(n-1)}\left(1+R_{x}^{(n)}\right) e^{-u_{n-1} h_{n-1}} .
\end{aligned}
$$

The transmission coefficients $A_{z(j+1)}, A_{z(j+k)}$ and $A_{z n}$, at the interfaces $j+1, j+k$, and $n$, are determined using the continuity condition expressions for $\hat{\hat{A}}_{v}$ (33):

$$
\begin{aligned}
A_{z(j+1)}= & \frac{2 A_{z j} \mathcal{Z}_{j} R_{z}^{(j+1)} e^{\lambda_{j} v_{j}\left(s_{1}-s_{2}\right)}}{\left(\mathcal{Z}_{j}-\mathcal{Z}_{j+1}\right)+\left(\mathcal{Z}_{j}+\mathcal{Z}_{j+1}\right) R_{z}^{(j+2)} e^{-2 \lambda_{j+1} v_{j+1} h_{j+1}}}, \\
A_{z(i+2)}= & \frac{A_{z(i+1)}\left(1+R_{z}^{(i+2)}\right) e^{-\lambda_{i+1} v_{i+1} h_{i+1}}}{1+R_{z}^{(i-3)} e^{-2 \lambda_{i+2} v_{i+2} h_{i+2}}} \\
& i=j, j+1, \ldots, j+k-2, \\
A_{z n}= & A_{z(n-1)}\left(1+R_{z}^{(n)}\right) e^{-\lambda_{n-1} v_{n-1} h_{n-1}} .
\end{aligned}
$$


The link between the solutions inside each homogeneous layer is given by the condition that the tangential components are continuous in the interfaces between the layers. This condition is enough to determine the reflection coefficients in equations 29 and 30. In order to write the coefficients $R_{x}^{(j)}, R_{x}^{(j+k)}, R_{z}^{(j)}$ and $R_{z}^{(j+k)}$, we define the surface impedance $\hat{\mathcal{Z}}$ and surface admittance $\hat{\mathcal{Y}}$, associated with the interfaces, in the $\left(k_{x}, k_{y}, z\right)$ domain as (Santos, 2007, in Portuguese):

$$
\hat{\mathcal{Z}}_{j-1}=-\left.\frac{\hat{\hat{E}}_{x, T M}^{(j)}}{\hat{\hat{H}}_{y, T M}^{(j)}}\right|_{z=z_{j}} ; \quad \hat{\mathcal{Z}}_{j+k}=\left.\frac{\hat{\hat{E}}_{x, T M}^{(j+k-1)}}{\hat{\hat{H}}_{y_{T M}}^{(j+k-1)}}\right|_{z=z_{j+k}} \quad k=1,2, \ldots, n-j .
$$

and

$$
\hat{\mathcal{Y}}_{j-1}=-\left.\frac{\hat{\hat{H}}_{y, T E}^{(j)}}{\hat{\hat{E}}_{x, T E}^{(j)}}\right|_{z=z_{j}} ; \quad \hat{\mathcal{Y}}_{j+k}=\left.\frac{\hat{\hat{H}}_{y, T E}^{(j+k-1)}}{\hat{\hat{E}}_{x, T E}^{(j+k-1)}}\right|_{z=z_{j+k}} \quad k=1,2, \ldots, n-j .
$$

Then the relations of the vector potential with the EM field (equations 6 and 22) give

$$
\begin{gathered}
\hat{\mathcal{Y}}_{j-1}=\left.\frac{\partial \frac{\partial \hat{\hat{A}}_{x(-)}^{(j)}}{\partial z}}{i \omega \mu_{j} \hat{\hat{A}}_{x(-)}^{(j)}}\right|_{z=z_{j}} \quad \text { and } \quad \hat{\mathcal{Y}}_{j+k}=-\left.\frac{\frac{\partial \hat{\hat{A}}_{x}^{(j+k-1)}}{\partial z}}{i \omega \mu_{j} \hat{\hat{A}}_{x}^{(j+k-1)}}\right|_{z=z_{j+k}} \quad k=1,2, \ldots, n-j . \\
\hat{\mathcal{Z}}_{j-1}=\left.\frac{\frac{1}{\sigma_{h j}} \frac{\partial \mathcal{A}_{v(-)}^{(j)}}{\partial z}}{\mathcal{A}_{v(-)}^{(j)}}\right|_{z=z_{j}} \quad \text { and } \quad \hat{\mathcal{Z}}_{j+k}=-\left.\frac{\frac{1}{\sigma_{h(j+k-1)}} \frac{\partial \mathcal{A}_{v}^{(j+k-1)}}{\partial z}}{\mathcal{A}_{v}^{(j+k-1)}}\right|_{z=z_{j+k}} \quad k=1,2, \ldots, n-j .
\end{gathered}
$$

By these definitions, the surface impedance and admittance at the last interface are simply the intrinsic impedance and admittance in the half-space. Then, starting from this last interface, the remaining surface parameters are given by the recurrence relations

$$
\begin{aligned}
& \hat{\mathcal{Z}}_{n}=\mathcal{Z}_{n}, \\
& \hat{\mathcal{Z}}_{i}=\mathcal{Z}_{i} \frac{\hat{\mathcal{Z}}_{i+1}+\mathcal{Z}_{i} \tanh \left[\lambda_{i} v_{i} h_{i}\right]}{\mathcal{Z}_{i}+\hat{\mathcal{Z}}_{i+1} \tanh \left[\lambda_{i} v_{i} h_{i}\right]}, \quad i=n-1, \ldots, j+1, \\
& \hat{\mathcal{Y}}_{n}=\mathcal{Y}_{n}, \\
& \hat{\mathcal{Y}}_{i}=\mathcal{Y}_{i} \frac{\hat{\mathcal{Y}}_{i+1}+\mathcal{Y}_{i} \tanh \left[u_{i} h_{i}\right]}{\mathcal{Y}_{i}+\hat{\mathcal{Y}}_{i+1} \tanh \left[u_{i} h_{i}\right]}, \quad i=n-1, \ldots, j+1 .
\end{aligned}
$$

Using these parameters, and applying the continuity condition to the solutions at the interfaces of layers $j$ and $j+k$ result in

$$
\begin{aligned}
R_{x}^{(j)}= & \frac{\left(\mathcal{Y}_{j}-\hat{\mathcal{Y}}_{j-1}\right)\left(\mathcal{Y}_{j}+\hat{\mathcal{Y}}_{j+1}\right)+\left(\mathcal{Y}_{j}-\hat{\mathcal{Y}}_{j-1}\right)\left(\mathcal{Y}_{j}-\hat{\mathcal{Y}}_{j+1}\right) e^{-2 u_{j} s_{2}}}{\left(\mathcal{Y}_{j}+\hat{\mathcal{Y}}_{j-1}\right)\left(\mathcal{Y}_{j}+\hat{\mathcal{Y}}_{j+1}\right)-\left(\mathcal{Y}_{j}-\hat{\mathcal{Y}}_{j-1}\right)\left(\mathcal{Y}_{j}-\hat{\mathcal{Y}}_{j+1}\right) e^{-2 u_{j} h_{j}}}, \\
R_{x}^{(j+1)}= & \frac{\left(\mathcal{Y}_{j}+\hat{\mathcal{Y}}_{j-1}\right)\left(\mathcal{Y}_{j}-\hat{\mathcal{Y}}_{j+1}\right)+\left(\mathcal{Y}_{j}-\hat{\mathcal{Y}}_{j-1}\right)\left(\mathcal{Y}_{j}-\hat{\mathcal{Y}}_{j+1}\right) e^{-2 u_{j} s_{1}}}{\left(\mathcal{Y}_{j}+\hat{\mathcal{Y}}_{j-1}\right)\left(\mathcal{Y}_{j}+\hat{\mathcal{Y}}_{j+1}\right)-\left(\mathcal{Y}_{j}-\hat{\mathcal{Y}}_{j-1}\right)\left(\mathcal{Y}_{j}-\hat{\mathcal{Y}}_{j+1}\right) e^{-2 u_{j} h_{j}}}, \\
R_{x}^{(j+k)}= & \frac{\mathcal{Y}_{j+k-1}-\hat{\mathcal{Y}}_{j+k}}{\mathcal{Y}_{j+k-1}+\hat{\mathcal{Y}}_{j+k}} \quad k=2,3, \ldots, n-j, \\
R_{z}^{(j)}= & \frac{\left(\mathcal{Z}_{j}-\hat{\mathcal{Z}}_{j-1}\right)\left(\mathcal{Z}_{j}+\hat{\mathcal{Z}}_{j+1}\right)-\left(\mathcal{Z}_{j}-\hat{\mathcal{Z}}_{j-1}\right)\left(\mathcal{Z}_{j}-\hat{\mathcal{Z}}_{j+1}\right) e^{-2 u_{j} s_{2}}}{\left(\mathcal{Z}_{j}+\hat{\mathcal{Z}}_{j-1}\right)\left(\mathcal{Z}_{j}+\hat{\mathcal{Z}}_{j+1}\right)-\left(\mathcal{Z}_{j}-\hat{\mathcal{Z}}_{j-1}\right)\left(\mathcal{Z}_{j}-\hat{\mathcal{Z}}_{j+1}\right) e^{-2 u_{j} h_{j}}}, \\
R_{z}^{(j+1)}= & \frac{\left(\mathcal{Z}_{j}+\hat{\mathcal{Z}}_{j-1}\right)\left(\mathcal{Z}_{j}-\hat{\mathcal{Z}}_{j+1}\right)-\left(\mathcal{Z}_{j}-\hat{\mathcal{Z}}_{j-1}\right)\left(\mathcal{Z}_{j}-\hat{\mathcal{Z}}_{j+1}\right) e^{-2 u_{j} s_{1}}}{\left(\mathcal{Z}_{j}+\hat{\mathcal{Z}}_{j-1}\right)\left(\mathcal{Z}_{j}+\hat{\mathcal{Z}}_{j+1}\right)-\left(\mathcal{Z}_{j}-\hat{\mathcal{Z}}_{j-1}\right)\left(\mathcal{Z}_{j}-\hat{\mathcal{Z}}_{j+1}\right) e^{-2 u_{j} h_{j}}}, \\
R_{z}^{(j+k)}= & \frac{\mathcal{Z}_{j+k-1}-\hat{\mathcal{Z}}_{j+k}}{\mathcal{Z}_{j+k-1}+\hat{\mathcal{Z}}_{j+k}} \quad k=2,3, \ldots, n-j .
\end{aligned}
$$


With the reflection coefficients in hand, the solutions for the vector potential in the $\left(k_{x}, k_{y}, z\right)$ domain is complete. Now the two final steps are: apply the double inverse Fourier transform to bring back the solution to the $(x, y, z)$ space, and calculate the electromagnetic field components from those of the vector potential using equations (6) and (22). The expressions are given in terms of double integrals in $k_{x}$ and $k_{y}$. Those are written in a more compact form if we apply the identity that relates the double Fourier transform with the Hankel transform, for problems of cylindrical symmetry, defining $k_{r}^{2}=k_{x}^{2}+k_{y}^{2}$ (Arfken, 1985, page 796):

$$
\frac{1}{2 \pi} \int_{-\infty}^{\infty} \int_{-\infty}^{\infty} f\left(k_{x}^{2}+k_{y}^{2}\right) e^{i\left(k_{x} x+k_{y} y\right)} d k_{x} d k_{y}=\int_{0}^{\infty} f\left(k_{r}\right) J_{0}\left(k_{r} r\right) k_{r} d k_{r} .
$$

For example, to find the solution for the electric field components in the layer with the source, at positions below it, we use the expressions for the $\hat{\hat{A}}_{x_{(+)}^{(j)}}^{(j)}$ and $\hat{\hat{A}}_{z_{(+)}}^{(j)}$ found in the equations (29) and (30). To write the integrals, use the kernel functions defined as

$$
\begin{aligned}
\mathcal{E}_{x y}^{h}\left(k_{r}\right) & =\frac{1}{\mathcal{Y}}\left(e^{-u_{j}\left(z-z_{j}-s_{1}\right)}+R_{x}^{(j)} e^{-u_{j}\left(z-z_{j}+s_{1}\right)}+R_{x}^{(j+1)} e^{u_{j}\left(z-z_{j}-s_{1}-2 s_{2}\right)}\right), \\
\mathcal{E}_{x y}^{v}\left(k_{r}\right) & =\lambda_{j} v_{j}\left[e^{-\lambda_{j} v_{j}\left(z-z_{j}-s_{1}\right)}-R_{z}^{(j)} e^{-\lambda_{j} v_{j}\left(z-z_{j}+s_{1}\right)}-R_{z}^{(j+1)} e^{\lambda_{j} v_{j}\left(z-z_{j}-s_{1}-2 s_{2}\right)}\right], \\
\mathcal{E}_{z}^{v}\left(k_{r}\right) & =\frac{1}{\sigma_{v j}}\left[e^{-\lambda_{j} v_{j}\left(z-z_{j}-s_{1}\right)}-R_{z}^{(j)} e^{-\lambda_{j} v_{j}\left(z-z_{j}+s_{1}\right)}+R_{z}^{(j+1)} e^{\lambda_{j} v_{j}\left(z-z_{j}-s_{1}-2 s_{2}\right)}\right] .
\end{aligned}
$$

Then the final expressions are

$$
\begin{aligned}
E_{x}^{(j)}(x, y, z)= & \frac{I(\omega) d s_{x}}{4 \pi \sigma_{h j}}\left(\frac{2 x^{2}}{r^{3}}-\frac{1}{r}\right) \int_{0}^{\infty} \mathcal{E}_{x y}^{v}\left(k_{r}\right) J_{1}\left(k_{r} r\right) d k_{r} \\
- & \frac{I(\omega) d s_{x}}{4 \pi \sigma_{h j}} \frac{x^{2}}{r^{2}} \int_{0}^{\infty} \mathcal{E}_{x y}^{v}\left(k_{r}\right) J_{0}\left(k_{r} r\right) k_{r} d k_{r} \\
+ & \frac{I(\omega) d s_{x}}{4 \pi}\left(\frac{2 y^{2}}{r^{3}}-\frac{1}{r}\right) \int_{0}^{\infty} \mathcal{E}_{x y}^{h}\left(k_{r}\right) J_{1}\left(k_{r} r\right) d k_{r} \\
- & \frac{I(\omega) d s_{x}}{4 \pi} \frac{y^{2}}{r^{2}} \int_{0}^{\infty} \mathcal{E}_{x y}^{h}\left(k_{r}\right) J_{0}\left(k_{r} r\right) k_{r} d k_{r} \\
E_{y}^{(j)}(x, y, z)= & \frac{I(\omega) d s_{x}}{2 \pi \sigma_{h j}} \frac{x y}{r^{3}} \int_{0}^{\infty} \mathcal{E}_{x y}^{v}\left(k_{r}\right) J_{1}\left(k_{r} r\right) d k_{r} \\
& -\frac{I(\omega) d s_{x}}{4 \pi \sigma_{h j}} \frac{x y}{r^{2}} \int_{0}^{\infty} \mathcal{E}_{x y}^{v}\left(k_{r}\right) J_{0}\left(k_{r} r\right) k_{r} d k_{r} \\
& -\frac{I(\omega) d s_{x}}{2 \pi} \frac{x y}{r^{3}} \int_{0}^{\infty} \mathcal{E}_{x y}^{h}\left(k_{r}\right) J_{1}\left(k_{r} r\right) d k_{r} \\
& +\frac{I(\omega) d s_{x}}{4 \pi} \frac{x y}{r^{2}} \int_{0}^{\infty} \mathcal{E}_{x y}^{h}\left(k_{r}\right) J_{0}\left(k_{r} r\right) k_{r} d k_{r}, \\
E_{z}^{(j)}(x, y, z)= & \frac{I(\omega) d s_{x}}{4 \pi} \frac{x}{r} \int_{0}^{\infty} \mathcal{E}_{z}^{v}\left(k_{r}\right) J_{1}\left(k_{r} r\right) k_{r}^{2} d k_{r} .
\end{aligned}
$$

In the same way, define the kernel functions for the expressions of the magnetic field:

$$
\begin{aligned}
\mathcal{H}_{x y}^{h}\left(k_{r}\right) & =\left[e^{-u_{j}\left(z-z_{j}-s_{1}\right)}+R_{x}^{(j)} e^{-u_{j}\left(z-z_{j}+s_{1}\right)}-R_{x}^{(j+1)} e^{u_{j}\left(z-z_{j}-s_{1}-2 s_{2}\right)}\right], \\
\mathcal{H}_{x y}^{v}\left(k_{r}\right) & =\left[e^{-\lambda_{j} v_{j}\left(z-z_{j}-s_{1}\right)}-R_{z}^{(j)} e^{-\lambda_{j} v_{j}\left(z-z_{j}+s_{1}\right)}+R_{z}^{(j+1)} e^{\lambda_{j} v_{j}\left(z-z_{j}-s_{1}-2 s_{2}\right)}\right], \\
\mathcal{H}_{z}^{h}\left(k_{r}\right) & =\frac{1}{u_{j}}\left[e^{-u_{j}\left(z-z_{j}-s_{1}\right)}+R_{x}^{(j)} e^{-u_{j}\left(z-z_{j}+s_{1}\right)}+R_{x}^{(j+1)} e^{u_{j}\left(z-z_{j}-s_{1}-2 s_{2}\right)}\right] .
\end{aligned}
$$


Then, the magnetic components are:

$$
\begin{aligned}
H_{x}^{(j)}(x, y, z)= & -\frac{I(\omega) d s_{x}}{2 \pi} \frac{x y}{r^{3}} \int_{0}^{\infty} \mathcal{H}_{x y}^{v}\left(k_{r}\right) J_{1}\left(k_{r} r\right) d k_{r} \\
& +\frac{I(\omega) d s_{x}}{4 \pi} \frac{x y}{r^{2}} \int_{0}^{\infty} \mathcal{H}_{x y}^{v}\left(k_{r}\right) J_{0}\left(k_{r} r\right) k_{r} d k_{r} \\
& +\frac{I(\omega) d s_{x}}{2 \pi} \frac{x y}{r^{3}} \int_{0}^{\infty} \mathcal{H}_{x y}^{h}\left(k_{r}\right) J_{1}\left(k_{r} r\right) d k_{r} \\
& -\frac{I(\omega) d s_{x}}{4 \pi} \frac{x y}{r^{2}} \int_{0}^{\infty} \mathcal{H}_{x y}^{h}\left(k_{r}\right) J_{0}\left(k_{r} r\right) k_{r} d k_{r}, \\
H_{y}^{(j)}(x, y, z)= & \frac{I(\omega) d s_{x}}{4 \pi}\left(\frac{2 x^{2}}{r^{3}}-\frac{1}{r}\right) \int_{0}^{\infty} \mathcal{H}_{x y}^{v}\left(k_{r}\right) J_{1}\left(k_{r} r\right) d k_{r} \\
- & \frac{I(\omega) d s_{x}}{4 \pi} \frac{x^{2}}{r^{2}} \int_{0}^{\infty} \mathcal{H}_{x y}^{v}\left(k_{r}\right) J_{0}\left(k_{r} r\right) k_{r} d k_{r} \\
+ & \frac{I(\omega) d s_{x}}{4 \pi}\left(\frac{2 y^{2}}{r^{3}}-\frac{1}{r}\right) \int_{0}^{\infty} \mathcal{H}_{x y}^{h}\left(k_{r}\right) J_{1}\left(k_{r} r\right) d k_{r} \\
- & \frac{I(\omega) d s_{x}}{4 \pi} \frac{y^{2}}{r^{2}} \int_{0}^{\infty} \mathcal{H}_{x y}^{h}\left(k_{r}\right) J_{0}\left(k_{r} r\right) k_{r} d k_{r}, \\
H_{z}^{(j)}(x, y, z) & =\frac{I(\omega) d s_{x}}{4 \pi} \frac{y}{r} \int_{0}^{\infty} \mathcal{H}_{z}^{h}\left(k_{r}\right) J_{1}\left(k_{r} r\right) k_{r}^{2} d k_{r}
\end{aligned}
$$

The solutions in equations (41) through (46) are valid for points located between the horizontal plane of the source and the sea-bed beneath it. To simulate mCSEM data, one simply has to make $z=0$ in the expressions.

All these integrals have to be evaluated numerically. To generate the results shown here, we have used the Fast Hankel Transform digital filter (Anderson, 1979).

To calculate the fields at any other position in the layers below or above the layer with the source, repeat the same steps, now using the appropriate equations for the vector potential as expressed in equations (29) and (30).

\section{APPLICATION}

Here we will investigate the effects of the anisotropy on the fields as they are measured in the marine CSEM method, considering the inline and broadside (or crossline) transmitter-receiver configurations. In all examples, the transmitter is at $30 \mathrm{~m}$ from the sea bottom, and the frequency is $0.25 \mathrm{~Hz}$.

First, we present results for the fields on the sea floor, to represent the measured data of the CSEM method, and analyse the influence of the anisotropy. Then the real and imaginary parts of the fields are viewed as vector plots, which allow for a geometrical analysis of the distribution of the field lines.

\section{CSEM data}

First let us consider a model of a homogeneous anisotropic halfspace under a $1500 \mathrm{~m}$ thick ocean layer (Fig. 2). In order to observe the effect of the anisotropy on CSEM data we calculate the fields in this model with different resistivity configurations for the half-space. In each case we compare the results obtained from the isotropic half-space with those obtained from the anisotropic case in two situations, with the vertical resistivity being two and then three times the value of the horizontal resistivity. In all cases, we take the isotropic case with $1.0 \Omega \cdot \mathrm{m}$ as a reference model in the comparisons.

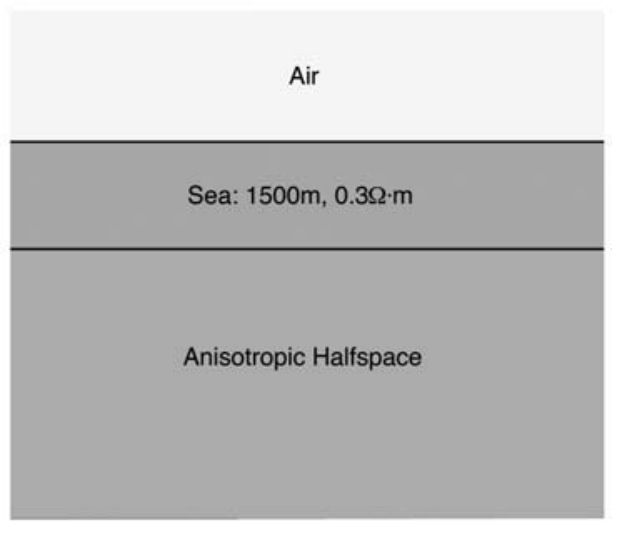

Figure 2 - Background model, without the resistive reservoir layer. 

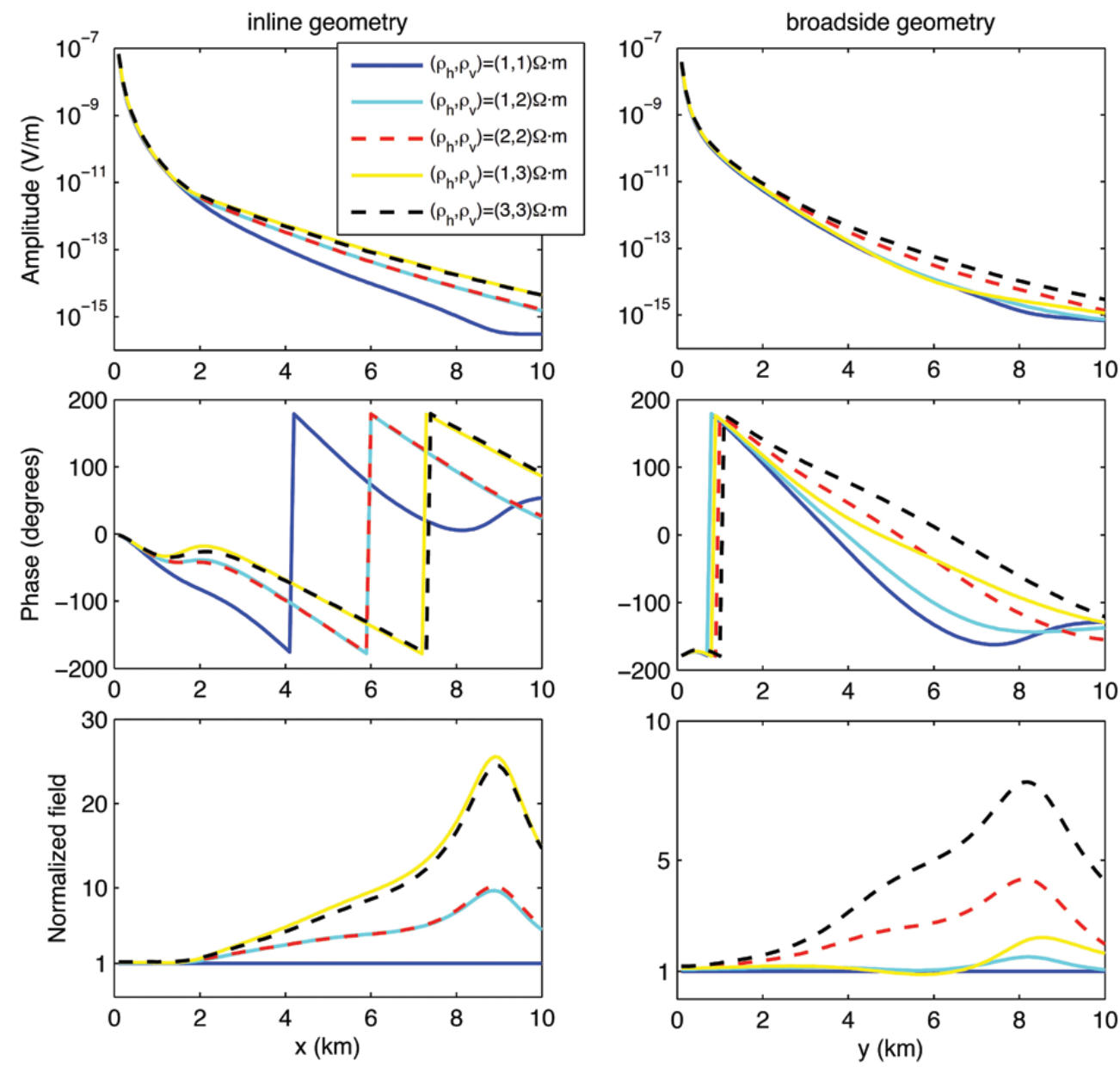

Figure 3 - Inline and broadside responses for $\boldsymbol{E}_{x}$, from the model of Figure 2.

Figure 3 shows that, for the measured inline $E_{x}$ component, the models with the same vertical resistivity present, approximately, the same response, even when the horizontal resistivity varies considerably. On the other hand, an increase in the value of the vertical resistivity implies a higher amplitude for the inline $E_{x}$ component. The broadside measurements indicate that the models with the same horizontal resistivity present the same approximate response for offsets up to $7 \mathrm{~km}$. At locations farther from the source, the responses begin to suffer influence from the vertical resistivity. The conclusions from these observations are that the inline $E_{x}$ component is more sensitive to the vertical resistivity, while the broadside $E_{x}$ component is considerably more sensitive to the horizontal resistivity at shorter offsets and shows an influence of both resistivity components at longer offsets. The analysis of the curves in Figure 4 shows that the same conclusions can be drawn for the $H_{y}$ component.

Figure 5 shows that the $E_{z}$ component is more sensitive to the vertical resistivity, while the $H_{z}$ component is affected only by the horizontal resistivity, as it is clear from the fact that the equation for this component (46), does not include $\sigma_{v}$.

Now, let us consider models in which there is included a resistive layer to represent a reservoir, as shown in Figure 6. In order to access the influence of the anisotropy on the "reservoir response", we analyse two studies: first, the reservoir layer is isotropic, with $100 \Omega \cdot \mathrm{m}$, and the host medium is anisotropic; second, both the reservoir and the host medium are anisotropic. In the first case, the anisotropic resistivity of the host medium varies to generate different responses (Fig. 6a), and in the second case, the resistivity of the host medium remains fixed, while the resistivity of the reservoir layer varies (Fig. 6b).

The responses from the model of Figure 6a, for varying the host's anisotropic resistivity, are shown in Figure 7 . Figures $7 a$ and $7 c$ show that a medium without a reservoir layer can generate an anomaly that's similar to the one from a medium with the reservoir layer, if the anisotropy of the host medium is underestimated. Therefore, a medium without a resistive layer could be 

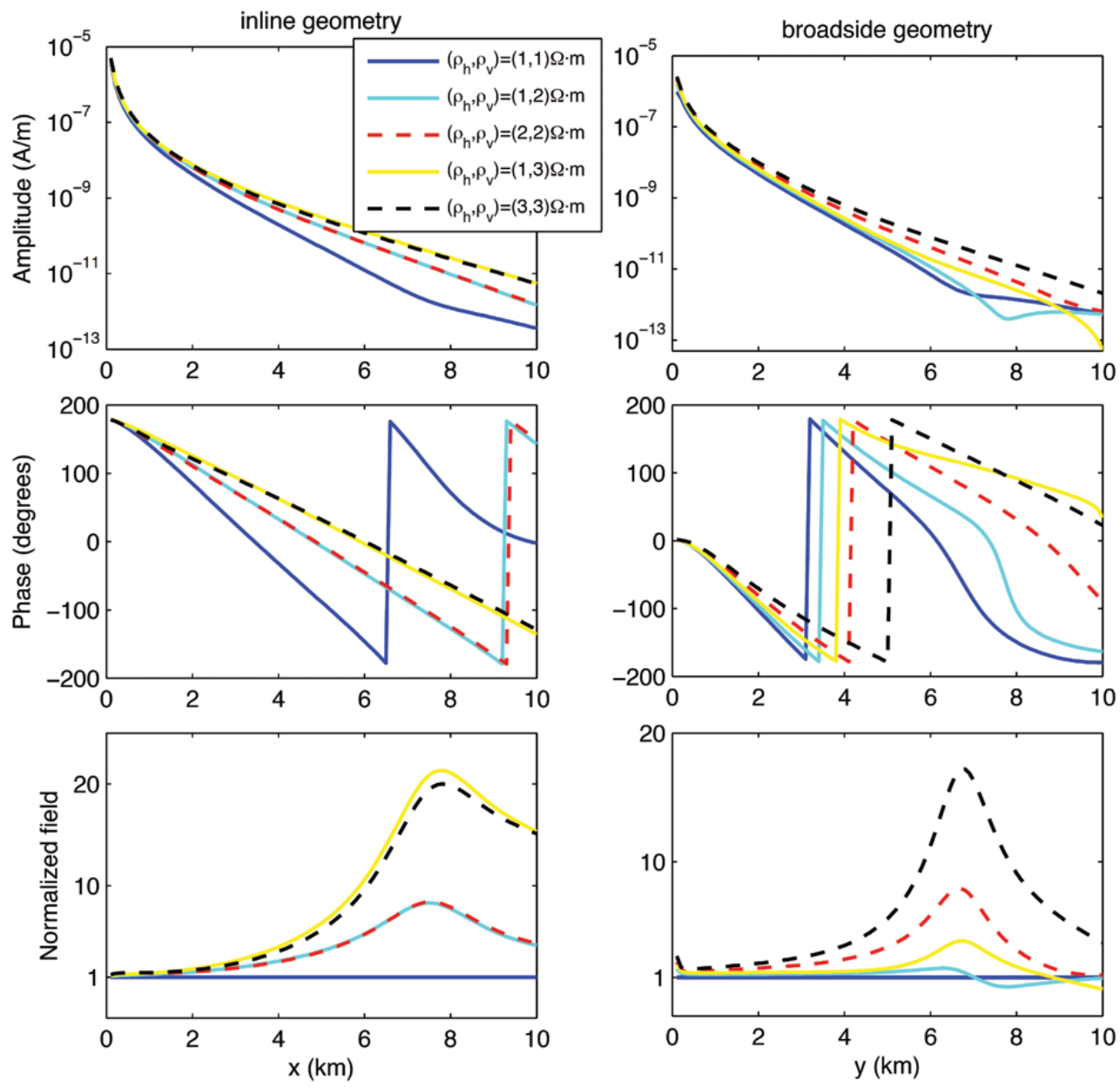

Figure 4 - Inline and broadside responses for $H_{y}$, from the model of Figure 2.

interpreted as containing a reservoir.

On the other hand, Figures $7 \mathrm{~b}$ and $7 \mathrm{~d}$ show that an overestimation of the anisotropy in the host medium minimizes the anomaly generated by the resistive reservoir layer. In this case, the incorrect interpretation might lead to an underestimation of the reservoir.

Figure 8 shows the response from the second simulation, with the fixed host resistivity and varying reservoir resistivity. In this situation, an increase in the vertical resistivity of the reservoir results in an increase in the amplitude of the $E_{x}$ component.

\section{Fields inside the layers}

This section presents an analysis of the distribution of the electric field and of the current density field, on the $x z$ plane, which contains the dipole source. The representation of the in phase and out of phase field components follows the style presented by
Um \& Alumbaugh (2007). Sections of the real and the imaginary parts of the frequency domain electric field and current density are shown as vector plots over a color representation of their respective amplitudes.

The study of these sections reinforces the conclusion that the inline data is most strongly influenced by the vertical resistivity. This fact is observed in Figures 9 to 12. Variations in the vertical resistivity produce considerable changes in the distribution of the electric field and the current density field, while the sections change very little when the vertical resistivity is kept the same while the horizontal resistivity varies.

The geometry of the field lines change when a resistive layer is added to the model, to represent a zone with a hydrocarbon accumulation. The comparison between the field distribution in the medium with and without the reservoir layer is made in Figures 13 and 14 . Notice that the difference between the two situations is greater in the isotropic models. In the anisotropic en- 

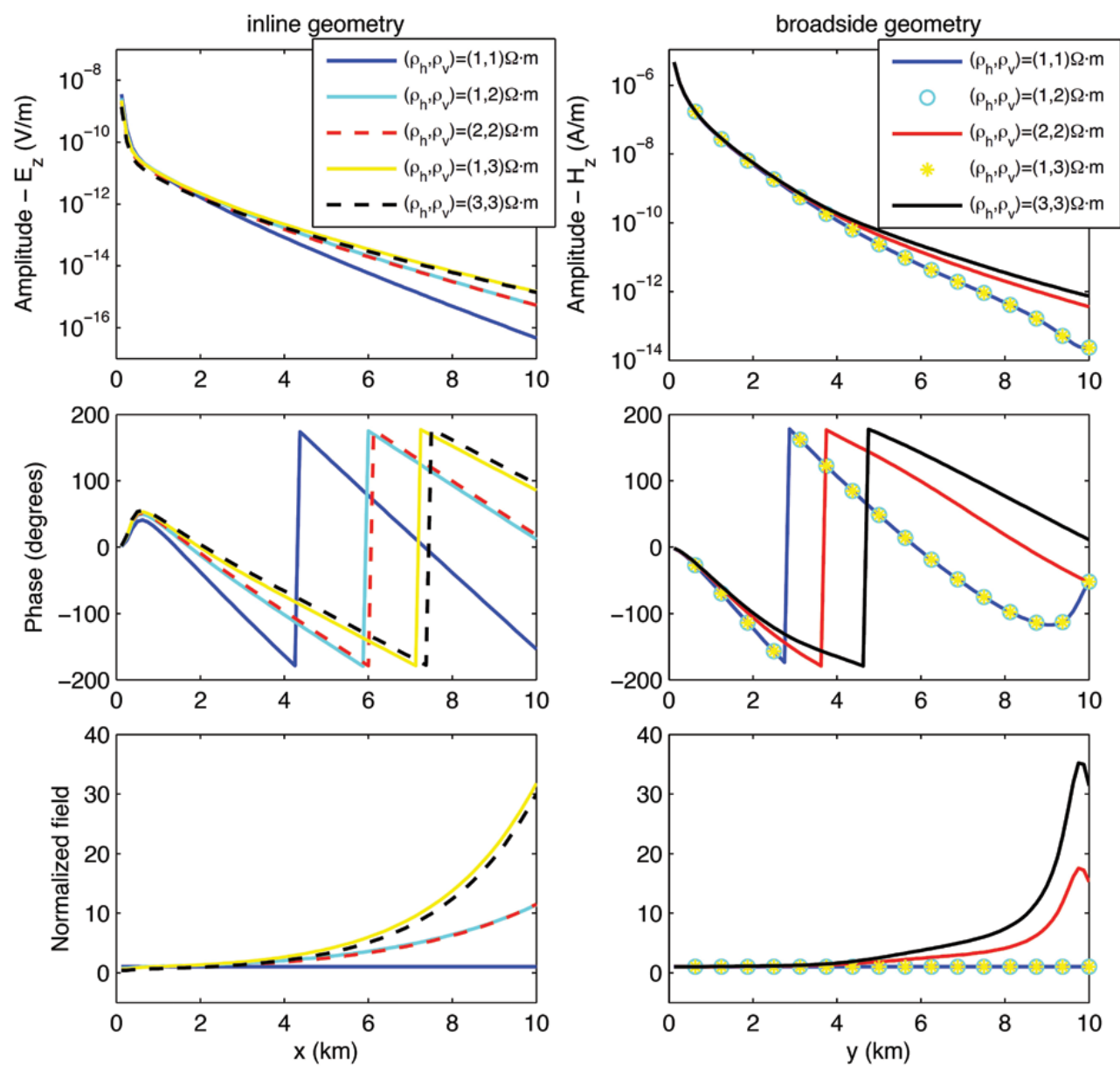

Figure $\mathbf{5}$ - Inline $\boldsymbol{E}_{z}$ and broadside $H_{z}$, from the model of Figure 2.

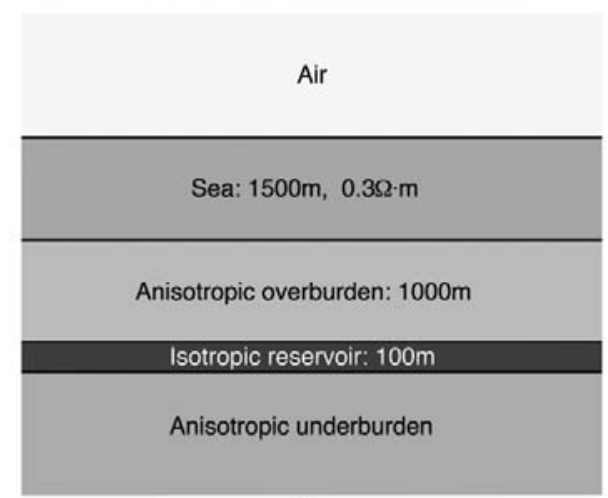

(a)

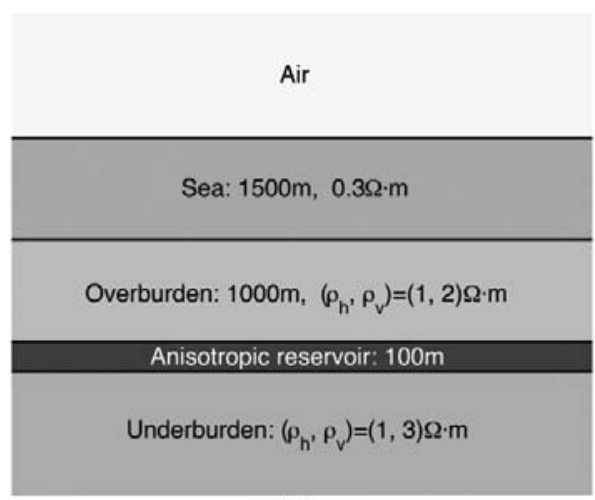

(b)

Figure 6 - Model with the resistive layer to represent the reservoir.

vironment, the geometry of the field lines is less changed by the presence of the resistive layer, particularly in the positions closer to the sea bed interface. The relatively small variation in the vertical resistivity of the background model ( $3 \Omega \cdot \mathrm{m}$, as compared with
$100 \Omega \cdot m$ in the reservoir layer) is enough to produce a significant change in the measured fields, which supports the conclusion that the anisotropy can in fact mask the presence of the reservoir, or at least make the data more difficult to be interpreted to identify it. 
anisotropic variations in resistivity of the background
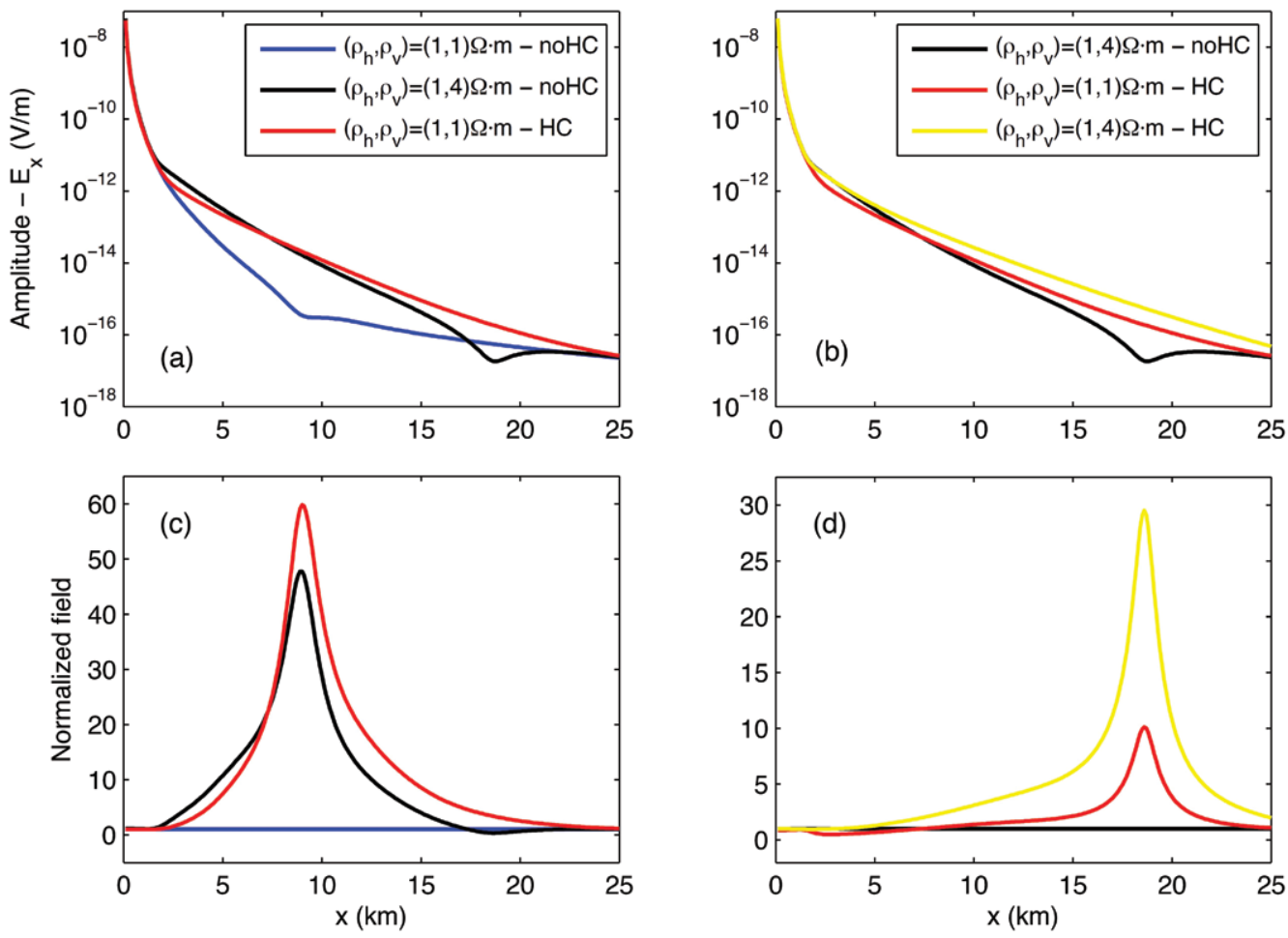

Figure 7 - Inline $E_{x}$ for the models of Figure 6a. In (c) the field is normalized by the values generated with $\left(\rho_{h}, \rho_{v}\right)=(1,1) \Omega \cdot m-$ noHC (blue line). In (d) the field is normalized by the values generated with $\left(\rho_{h}, \rho_{v}\right)=(1,4) \Omega \cdot m-$ noHC (black line).
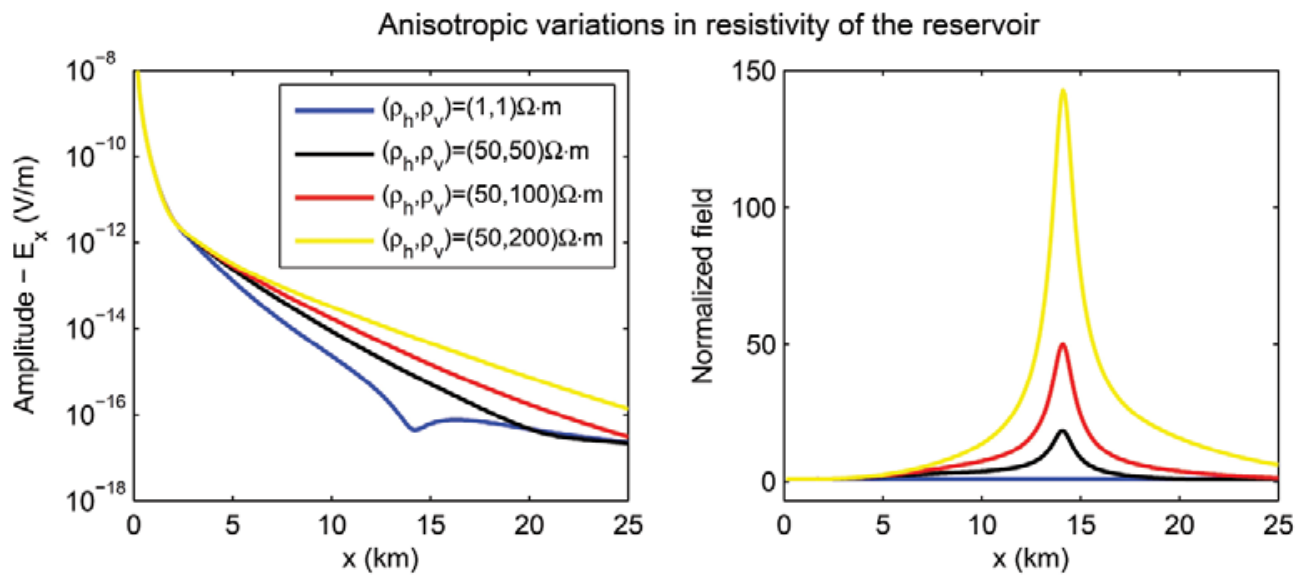

Figure 8 - Inline $E_{x}$ from the models of Figure $6 b$.

\section{CONCLUSIONS}

The calculation shown here follows a traditional approach to problems of dipole sources. The use of the vector potential and of the Fourier and Hankel transforms yield a semi-analytical solution, in terms of Bessel integrals, which are easily evaluated with digital filters, or other techniques.
In our view, the detailed step-by-step presentation is particularly useful for the student or for the beginner in this kind of problem, first because it helps clarify the solutions and allows a better grasp of the problem, but also because of the general aspects of such a method, which can be implemented to different sources of interest in geophysical problems. 

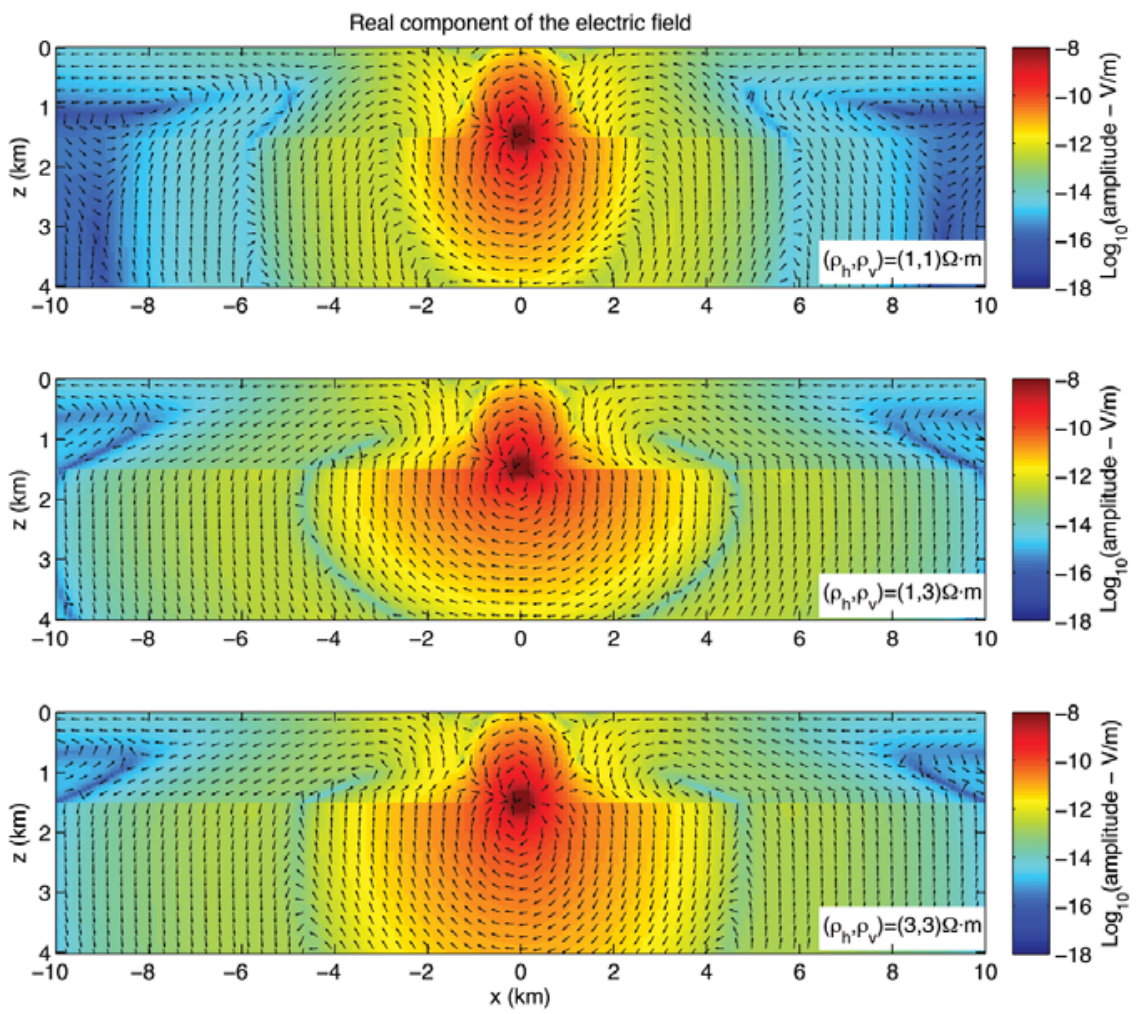

Figure 9 - Real component of the electric field in the $x z$ plane for the model without the reservoir.
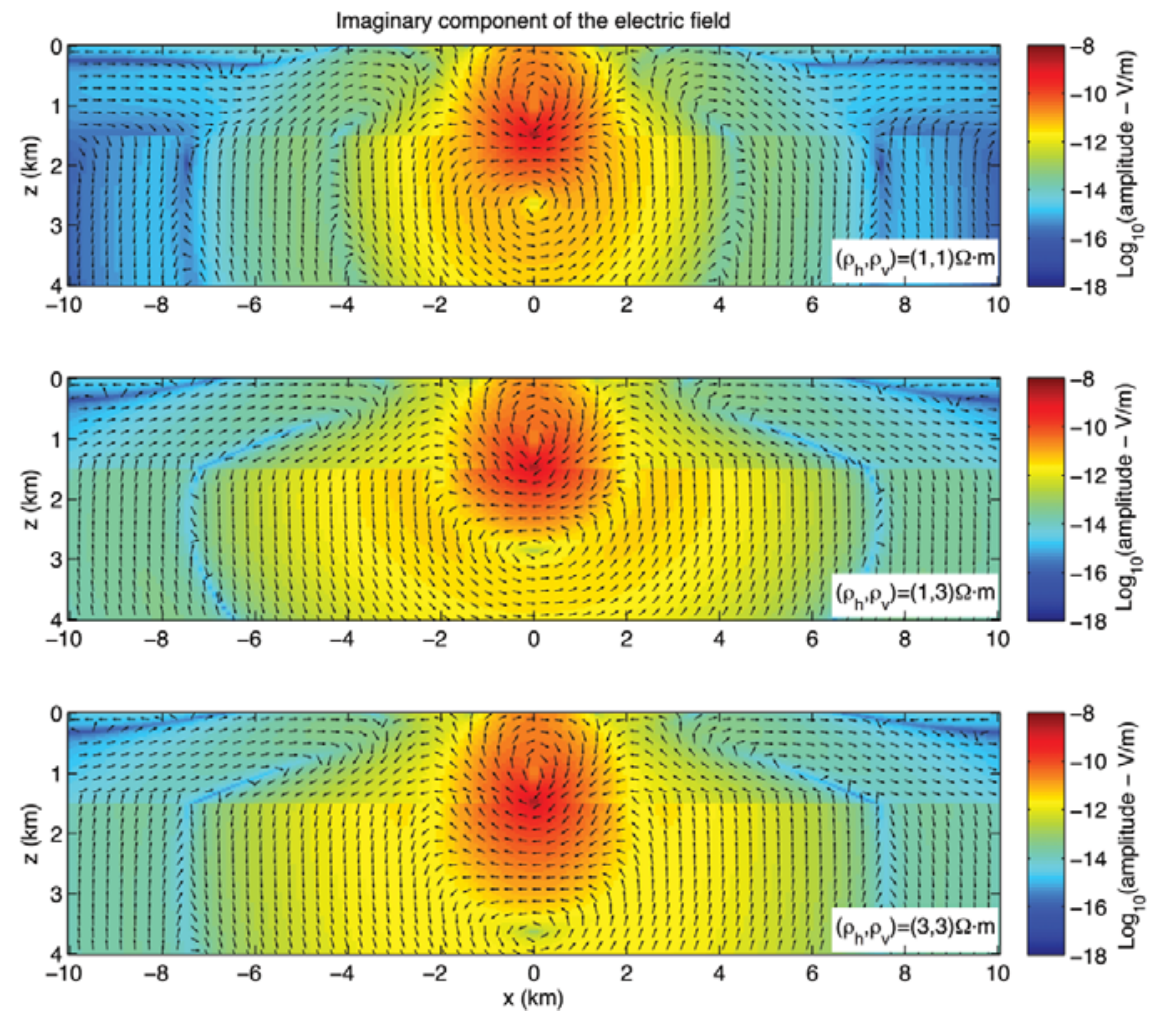

Figure 10 - Imaginary component of the electric field in the $x z$ plane for the model without the reservoir. 

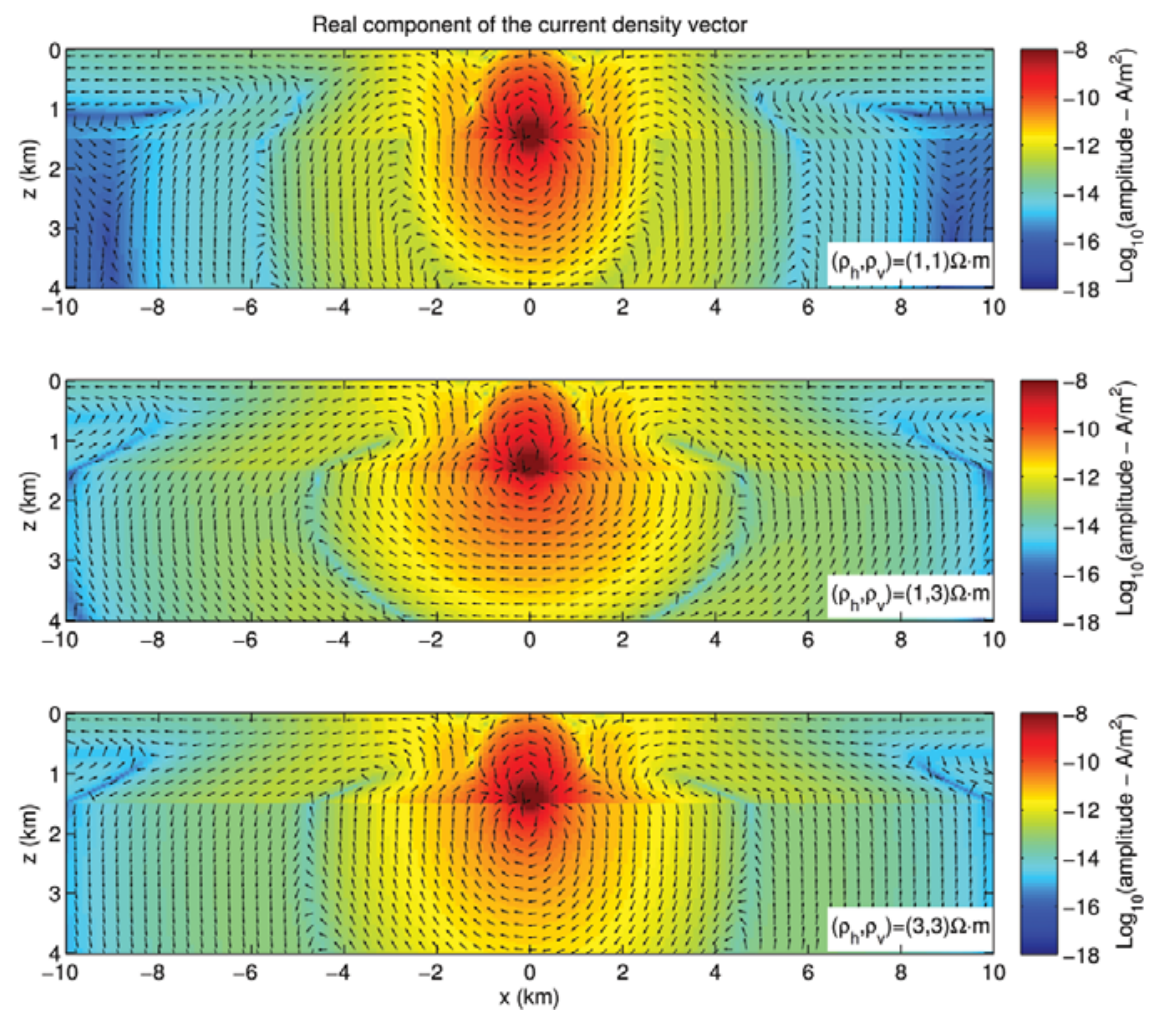

Figure 11 - Real component of the current density vector field in the $x z$ plane for the model without the reservoir.
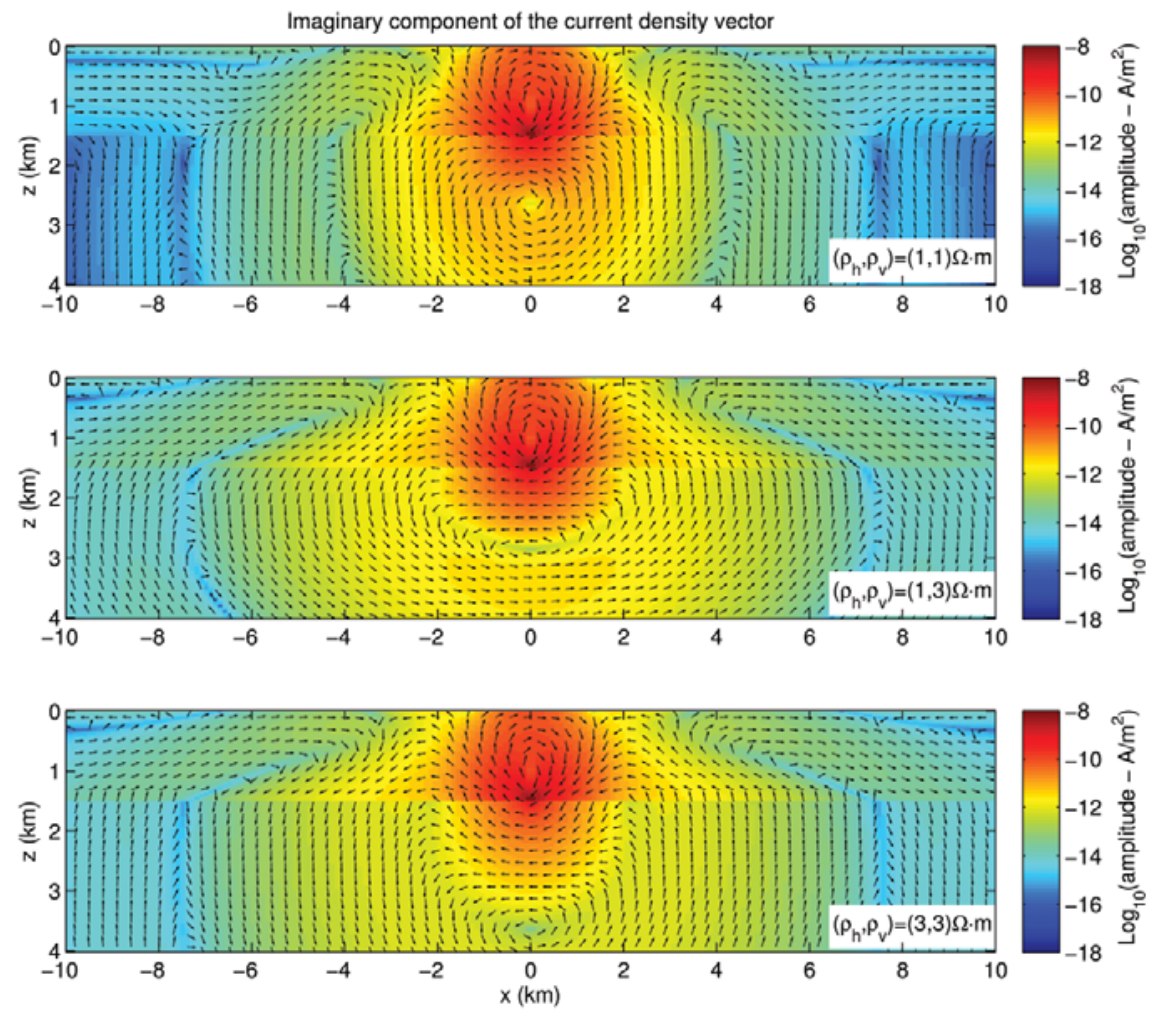

Figure 12 - Imaginary component of the current density vector field in the $x z$ plane for the model without the reservoir. 

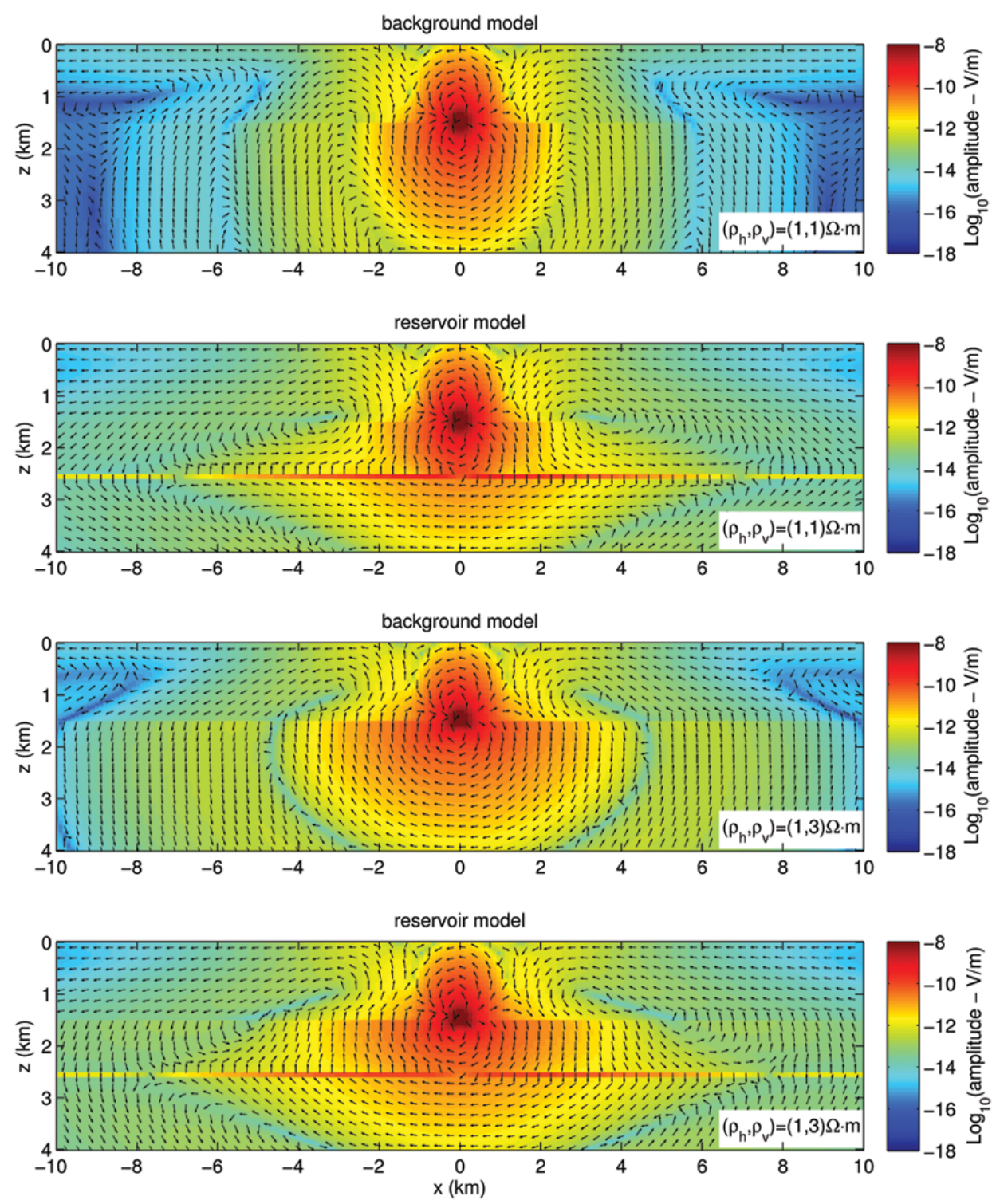

Figure 13 - Comparison of the real component of the electric field in the $x z$ plane in the models with and without the reservoir.

\section{ACKNOWLEDGEMENTS}

The authors thank the support given by the National Institute of Science and Technology of Petroleum Geophysics - INCT/GP and by the National Council for the Scientific and Technological Development - CNPq. Régis is recipient of the CNPq research scholarship.

\section{REFERENCES}

ANDERSON WL. 1979. Numerical integration of related Hankel transforms of orders 0 and 1 by adaptive digital filtering. Geophysics, 44: $1287-1305$.
ARFKEN G. 1985. Mathematical methods for physicists. 3rd ed., Academic Press. 985 pp.

CONSTABLES \& SRNKA L. 2007. An introduction to marine controlledsource electromagnetic methods for hydrocarbon exploration. Geophysics, 72: WA3-WA12.

KAUFMANA \& DASHEVSKY YA. 2003. Principles of Induction Logging. Elsevier, volume 38 of "Methods in Geochemistry and Geophysics", 11: 605-625.

KONG FN, JOHNSTAD SE, RØSTEN T \& WESTERDAHL H. 2008. A 2.5D finite-element-modeling difference method for marine CSEM modeling in stratified anisotropic media. Geophysics, 73: F9-F19. 

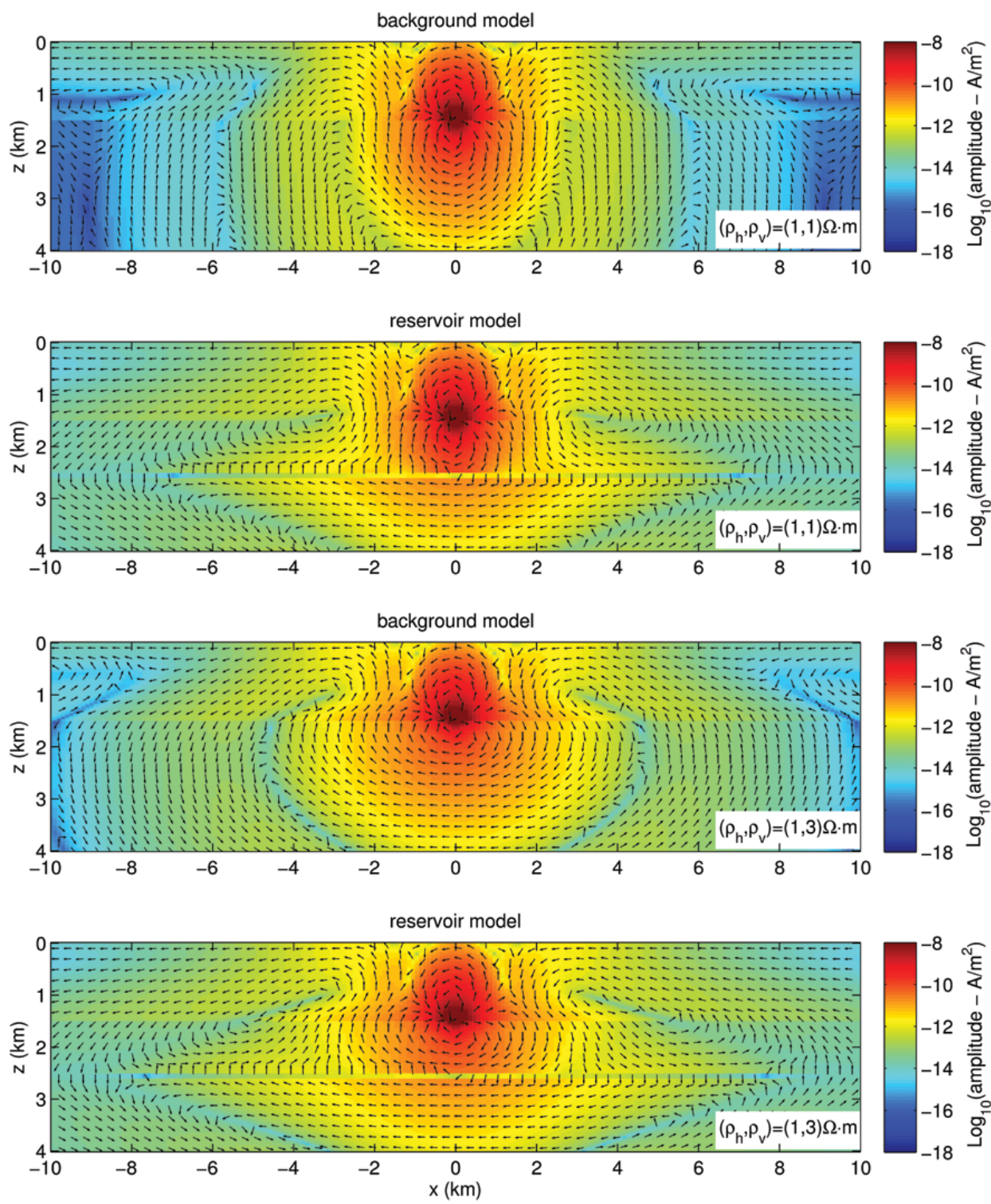

Figure 14 - Comparison of the real component of the current density vector field in the $x z$ plane in the models with and without the reservoir.

KONG JA. 1990. Electromagnetic wave theory. 2nd ed., John Wiley \& Sons, Inc. 667 pp.

LI Y \& DAI S. 2011. Finite element modelling of marine controlled-source electromagnetic responses in two-dimensional dipping anisotropic conductivity structures. Geophysical Journal International, 185: 622636.

LØSETHLO \& URSIN B. 2007. Electromagnetic fields in planarly layered anisotropic media. Geophysical Journal International, 170: 44-80.

LU X \& XIA C. 2007. Understanding anisotropy in marine CSEM data. In: SEG Technical Program Expanded Abstracts 2007; 77th Annual Meeting, San Antonio, TX. Society of Exploration Geophysicists, 633637.
RAMANANJAONA C \& MacGREGOR L. 2010. 2.5D inversion of CSEM data in a vertically anisotropic earth. Journal of Physics: Conference Series, 255: 012004.

SANTOS WG. 2007. Arranjos coplanar e coaxial nas sondas de perfilagem de poço: Ferramentas triaxiais em reservatórios laminados. Master's thesis, Universidade Federal do Pará, Brazil. 70 pp.

SPIES BR \& FRISCHKNECHT FC. 1987. Electromagnetic Sounding. In: NABIGHIAN MN (Ed.). Electromagnetic Methods in Applied Geophysics; SEG, volume 2 of "Investigations in Geophysics", 5: 285-425.

TOMPKINS M. 2005. The role of vertical anisotropy in interpreting marine controlled-source electromagnetic data. In: SEG Technical Program 
Expanded Abstracts 2005; 75th Annual Meeting, Houston, TX. Society of Exploration Geophysicists, 514-517.

UM E \& ALUMBAUGHD. 2007. On the physics of the marine controlledsource electromagnetic method. Geophysics, 72: WA13-WA26.

WAIT JR. 1981. Wave propagation theory. Pergamon Press. 348 pp. Elmsford, New York.
WARD SH \& HOHMANN GW. 1987. Electromagnetic Theory for Geophysical Applications. In: NABIGHIAN MN (Ed.). Electromagnetic Methods in Applied Geophysics; SEG, volume 1 of Investigations in Geophysics, 4: 130-311.

XIONG Z. 1989. Electromagnetic fields of electric dipoles embedded in a stratified anisotropic earth. Geophysics, 54: 1643-1646.

Recebido em 6 maio, 2014 / Aceito em 17 julho, 2015

Received on May 6, 2014 / Accepted on July 17, 2015

\section{NOTES ABOUT THE AUTHORS}

Walleson Gomes dos Santos undergraduated both in Mathematics and Physics in 2004. Obtained M.Sc. in Borehole Geophysics from the Universidade Federal do Pará, Brazil, in 2007, and a doctorate in Geophysical Electromagnetic Methods in 2014. Teaches undergraduate Physics and Mathematics at the Centro de Instrução Almirante Braz de Aguiar, in Belém, Brazil.

Cícero Roberto Teixeira Régis got a doctorate in Geophysics from the Universidade Federal do Pará, UFPA, Brazil, in 2000. Associate Professor of the Faculty of Geophysics in the UFPA since 2004. Recipient of a Research Scholarship from the CNPq. Research includes the numerical modeling and inversion of electromagnetic geophysical methods. 